Bull. Korean Math. Soc. 51 (2014), No. 3, pp. 691-700

http://dx.doi.org/10.4134/BKMS.2014.51.3.691

\title{
NORMAL FAMILY OF MEROMORPHIC FUNCTIONS
}

\author{
JIAN-PING WANG
}

ABstract. We study normality for families of meromorphic functions which is related to an extended version of a Hayman's conjecture on value distribution, and prove several normality criteria for meromorphic functions and certain non-homogeneous differential polynomials.

\section{Introduction and results}

We shall use the usual notations and classical results of Nevanlinna's theory (see [16]). Let $f, g$ be non-constant meromorphic functions and $c$ be a finite complex number. We say that $f$ and $g$ share the value $c$ if $f-c$ and $g-c$ have the same zeros (see $[16])$. Throughout the paper, we denote by $\mathbb{C}$ the complex plane and by $D$ a domain in $\mathbb{C}$.

A family $\mathcal{F}$ of functions meromorphic in $D$ is said to be normal if each sequence in $\mathcal{F}$ has a subsequence which converges spherically uniformly on compact subsets of $D[12]$.

Hayman [7] proposed a well-known conjecture on value distribution: If $f$ is a transcendental meromorphic functions and $n$ is a positive integer, then $f^{n} f^{\prime}$ assumes every finite non-zero value infinitely often. This conjecture, following partial results by Clunie [5], Mues [10] and Hayman [7], was finally confirmed by Bergweiler and Eremenko [2], Chen and Fang [4], independently.

In 1993, C. C. Yang, L. Yang and Y. F. Wang [15] considered an extended version of the above Hayman's conjecture and proved that if $f$ is a transcendental entire function, and $k, n(\geq 2)$ are positive integers, then $f\left(f^{(k)}\right)^{n}$ assumes every finite non-zero value infinitely often. They also pointed out, but without proof, that the same conclusion holds for $n=1$. Although the current partial answers to this Yang's problem are affirmative (see $[1,3,8,9,11,13,14,18]$ ), the verification of its validity may need more time.

Received February 19, 2013.

2010 Mathematics Subject Classification. 30D35, 30D45.

Key words and phrases. meromorphic function, normal family, differential polynomial.

This work was supported by the National Natural Science Foundation of China (Grant No.11041005) and the Natural Science Foundation of Zhejiang Province, China (Grant No.Y6090641). 
According to Bloch's principle (see [17, p. 222]), it is natural to consider the normality for family of meromorphic functions corresponding to the above Yang's problem. In this direction, Pang and Zalcman proved the following results.

Theorem A ([11]). Let $k$ be a positive integer and let $\mathcal{F}$ be a family of functions holomorphic in a unit disc $\Delta$ such that each $f \in \mathcal{F}$ has only zeros of multiplicity at least $k$. Suppose that there exist positive integer $n$ and finite non-zero complex number $c$ such that $f^{n} f^{(k)} \neq c$ for each $f \in \mathcal{F}$ and all $z \in \Delta$. Then $\mathcal{F}$ is normal in $\Delta$.

In this paper, we study the normality for families of meromorphic functions corresponding to the above Yang's problem and prove the following results.

Theorem 1. Let $k \geq 3$ be a positive integer and let $c$ be a finite non-zero complex number. Let $\mathcal{F}$ be a family of functions meromorphic in $D$ such that each $f \in \mathcal{F}$ has only zeros of multiplicity at least $k$ and poles of multiplicity at least 2. If, for each pair of functions $f$ and $g$ in $\mathcal{F}, f f^{(k)}$ and $g g^{(k)}$ share the value $c$, then $\mathcal{F}$ is normal in $D$.

Example. Let $D=\{z:|z|<1\}$ and $\mathcal{F}=\left\{f_{j}\right\}$, where $f_{j}(z)=j z^{k-1}, z \in$ $D, j=1,2, \ldots$. We see that each $f_{j} \in \mathcal{F}$ has only zero of multiplicity $k-1$ and that $f_{j} f_{j}^{(k)}$ and $f_{l} f_{l}^{(k)}$ share $c$ for each pair of functions $f_{j}$ and $f_{l}$ in $\mathcal{F}$. But $\mathcal{F}$ is not normal at $z=0$.

The above example shows that the hypothesis that each $f \in \mathcal{F}$ has only zeros of multiplicity at least $k$ is best possible for Theorem 1 .

For normality criteria concerning differential polynomials, we have:

Theorem 2. Let $k \geq 3$ be a positive integer and let $a_{1}(z), a_{2}(z), \ldots, a_{k+2}(z)$ be functions holomorphic in $D$ with $a_{k+2}(z) \neq 0$. Let $\mathcal{F}$ be a family of functions meromorphic in $D$ such that each $f \in \mathcal{F}$ has only zeros of multiplicity at least $k$ and poles of multiplicity at least 2 . Set

$$
E(f)=\left\{z \in D: \sum_{m=0}^{k} a_{m}(z) f(z) f^{(k-m)}(z)+a_{k+1}(z) f(z)+a_{k+2}(z)=0\right\},
$$

where $a_{0}(z) \equiv 1$ and $f^{(0)} \equiv f$. If there exists a constant $M>0$ such that $\left|f^{(k)}(z)\right| \leq M$ for each $f \in \mathcal{F}$ and all $z \in E(f)$, then $\mathcal{F}$ is normal in $D$.

Suppose that $a_{m}(z) \equiv 0$ for $m=1,2, \ldots, k+1$. Then the following corollaries are immediate results of Theorem 2 .

Corollary 1. Let $k \geq 3$ be a positive integer and let $c(z)$ be a non-vanishing holomorphic function in $D$. Let $\mathcal{F}$ be a family of functions meromorphic in $D$ such that each $f \in \mathcal{F}$ has only zeros of multiplicity at least $k$ and poles of multiplicity at least 2. If $f(z) f^{(k)}(z) \neq c(z)$ for each $f \in \mathcal{F}$ and all $z \in D$, then $\mathcal{F}$ is normal in $D$. 
Corollary 2. Let $k \geq 3$ be a positive integer and let $c(z)$ be a non-vanishing holomorphic function in $D$. Let $\mathcal{F}$ be a family of functions holomorphic in $D$ such that each $f \in \mathcal{F}$ has only zeros of multiplicity at least $k$. If $f(z) f^{(k)}(z) \neq$ $c(z)$ for each $f \in \mathcal{F}$ and all $z \in D$, then $\mathcal{F}$ is normal in $D$.

Obviously Corollary 2 generalizes Theorem A from a non-zero constant $c$ to a function $c(z)$ which is holomorphic and non-vanishing in $D$ for the cases $k \geq 3$ and $n=1$.

\section{Lemmas}

Lemma 1 ([17, p. 216]). Let $\mathcal{F}$ be a family of functions meromorphic in the unit disc $\triangle$, let $k, l$ be positive integers and $\alpha$ be a real number with $-l<\alpha<k$. Suppose that all zeros of functions in $\mathcal{F}$ have multiplicity at least $k$ and all poles of functions in $\mathcal{F}$ have multiplicity at least $l$ and that $\mathcal{F}$ is not normal at $z_{0} \in \triangle$. Then there exist functions $f_{j} \in \mathcal{F}$, points $z_{j} \in \triangle$, positive numbers $\rho_{j}$ and $a$ non-constant function $g$ which is meromorphic in $\mathbb{C}$ such that $z_{j} \rightarrow z_{0}, \rho_{j} \rightarrow 0$ and $\rho_{j}^{-\alpha} f\left(z_{j}+\rho_{j} \zeta\right) \rightarrow g(\zeta)$ spherically uniformly on compact sets of $\mathbb{C}$.

Lemma 2 ([6, Lemma 2$])$. Let $f$ be a non-constant meromorphic function and let $Q_{1}[f], Q_{2}[f]$ be differential polynomials in $f$. Let $n$ be a positive integer and $f^{n} Q_{1}[f]=Q_{2}[f]$. If $\gamma_{Q_{2}} \leq n$, then $m\left(r, Q_{1}[f]\right)=S(r, f)$, where $\gamma_{Q_{2}}$ is the degree of $Q_{2}[f]$.

To state our lemmas, we need the following notations.

Let $f$ be a non-constant meromorphic function in $\mathbb{C}$ and let $k$ be a positive integer. We denote by $N_{k}(r, 1 / f)$ the counting function for zeros of $f$ with multiplicity at most $k, N_{(k}(r, 1 / f)$ the counting function for zeros of $f$ with multiplicity at least $k$, and $N_{k}(r, 1 / f)$ the counting function for zeros of $f$ with multiplicity $k$. As usual, we use $\bar{N}_{k)}(r, 1 / f), \bar{N}_{(k}(r, 1 / f)$ and $\bar{N}_{k}(r, 1 / f)$ to denote the corresponding reduced ones, without regard to multiplicity.

Lemma 3. Let $k \geq 3$ be a positive integer and let $f$ be a transcendental meromorphic function in $\mathbb{C}$. If $f$ has only zeros of multiplicity at least $k$ and poles of multiplicity at least 2 , then $f f^{(k)}$ assumes every finite non-zero complex number infinitely often.

Proof. Let $c$ be a finite non-zero complex number. Set

$$
F=f f^{(k)}-c .
$$

By (2.1) we have

$$
T(r, F)=O(T(r, f)) .
$$

Rewriting (2.1) as $F-f f^{(k)}=-c$, which leads to

$$
(-c) \frac{F^{\prime}}{F}=\left(F-f f^{(k)}\right) \frac{F^{\prime}}{F}=F^{\prime}-f f^{(k)} \frac{F^{\prime}}{F}=f^{\prime} f^{(k)}+f f^{(k+1)}-f f^{(k)} \frac{F^{\prime}}{F},
$$


so that

$$
f \phi=(-c) \frac{F^{\prime}}{F}
$$

where

$$
\phi=\frac{f^{\prime}}{f} f^{(k)}+f^{(k+1)}-f^{(k)} \frac{F^{\prime}}{F} .
$$

We shall show that $F$ is not a constant. Otherwise we have $f f^{(k)} \equiv b$ for some constant $b$. Since $f$ has only zeros of multiplicity at least $k$, we find $b \neq 0$ and thus $f \neq 0$, which means that $b / f^{2}\left(\equiv f^{(k)} / f\right)$ must be an entire function. This together with Nevanlinna's first fundamental theorem yields

$$
2 T(r, f)+O(1)=T\left(r, b / f^{2}\right)=m\left(r, b / f^{2}\right)=m\left(r, f^{(k)} / f\right)=S(r, f),
$$

so that $f$ is a constant. It is impossible. Hence $F$ is not a constant.

Now we can derive from (2.3) that $\phi \not \equiv 0$. By applying Lemma 2 to $(2.3)$ and noting (2.2) we obtain

$$
m(r, \phi)=S(r, f) .
$$

From (2.1) we see that any pole of $f$ must be a simple pole of $F^{\prime} / F$, which and (2.3) means that any pole of $f$ with multiplicity $m(\geq 2)$ must be a zero of $\phi$ with multiplicity $m-1$. Thus we have

$$
N_{(2}(r, f) \leq N(r, 1 / \phi)+\bar{N}(r, 1 / \phi) .
$$

If $z_{0}$ is a zero of $f$ with multiplicity $n(\geq k+1)$, then we see from (2.1) that $F^{\prime}$ has zeros at $z_{0}$ with multiplicity at least $n$ and, so is $F^{\prime} / F$ simply noting $F\left(z_{0}\right)=-c$. From this and (2.3) it follows that $z_{0}$ will never be a pole of $\phi$. Therefore, we deduce from (2.4) that $\phi$ can only have poles at the zeros of $F$ and the zeros of $f$ with multiplicity at most $k$. This and (2.4) gives

$$
N(r, \phi) \leq \bar{N}_{k)}(r, 1 / f)+\bar{N}(r, 1 / F)
$$

From (2.2),(2.3) and Nevanlinna's first fundamental theorem, we have

$$
m(r, f) \leq m(r, 1 / \phi)+S(r, f)=T(r, \phi)-N(r, 1 / \phi)+S(r, f) .
$$

Since $f$ has no simple poles, from (2.5)-(2.8) we obtain

$$
\begin{aligned}
T(r, f) & =m(r, f)+N_{(2}(r, f) \leq T(r, \phi)+\bar{N}(r, 1 / \phi)+S(r, f) \\
& \leq 2 T(r, \phi)+S(r, f) \leq 2 \bar{N}_{k)}(r, 1 / f)+2 \bar{N}(r, 1 / F)+S(r, f) .
\end{aligned}
$$

Noting that $f$ has only zeros with multiplicity at least $k$, thus we have

$$
\bar{N}_{k)}(r, 1 / f)=\bar{N}_{k}(r, 1 / f)=\frac{1}{k} N_{k}(r, 1 / f) \leq \frac{1}{k} T(r, f)+O(1) .
$$

From (2.9) and (2.10) we obtain

$$
\left(1-\frac{2}{k}\right) T(r, f) \leq 2 \bar{N}(r, 1 / F)+S(r, f),
$$

so that $F$ has infinitely many zeros since $k \geq 3$. Lemma 3 is proved. 
Lemma 4. Let $k \geq 2$ be a positive integer and let $c$ be a non-zero constant. Suppose that $f$ is a rational function but not a polynomial and that $f$ has only zeros of multiplicity at least $k$ and poles of multiplicity at least 2 , then $f f^{(k)}-c$ has at least two distinct zeros.

Proof. If, to the contrary, $f f^{(k)}-c$ has at most one zero. We set

$$
f=\frac{\alpha\left(z-a_{1}\right)^{m_{1}}\left(z-a_{2}\right)^{m_{2}} \cdots\left(z-a_{s}\right)^{m_{s}}}{\left(z-b_{1}\right)^{n_{1}}\left(z-b_{2}\right)^{n_{2}} \cdots\left(z-b_{t}\right)^{n_{t}}}=\frac{Q(z)}{P(z)}, \text { say, }
$$

where $a_{\mu}(\mu=1,2, \ldots, s), b_{\nu}(\nu=1,2, \ldots, t)$ and $\alpha(\neq 0)$ are constants, $P(z)$ and $Q(z)$ are relatively prime polynomials. By the assumptions we have $m_{\mu} \geq$ $k(\mu=1,2, \ldots, s)$ and $n_{\nu} \geq 2(\nu=1,2, \ldots, t)$. For simplicity we write

$$
\begin{gathered}
q=m_{1}+m_{2}+\cdots+m_{s} \geq k s, \\
p=n_{1}+n_{2}+\cdots+n_{t} \geq 2 t .
\end{gathered}
$$

By differentiating (2.11) $k$ times we have

$$
f^{(k)}=\frac{\alpha\left(z-a_{1}\right)^{m_{1}-k}\left(z-a_{2}\right)^{m_{2}-k} \cdots\left(z-a_{s}\right)^{m_{s}-k} G(z)}{\left(z-b_{1}\right)^{n_{1}+k}\left(z-b_{2}\right)^{n_{2}+k} \cdots\left(z-b_{t}\right)^{n_{t}+k}},
$$

where $G(z)$ is a polynomial of degree at most $k(s+t-1)$, with constants as coefficients. In fact we have

$G(z)=(q-p)(q-p-1) \cdots[q-p-(k-1)] z^{k(s+t-1)}+d_{1} z^{k(s+t-1)-1}+\cdots+d_{k(s+t-1)}$.

From (2.11) and (2.14) we get

$$
f f^{(k)}=\frac{\alpha^{2}\left(z-a_{1}\right)^{2 m_{1}-k}\left(z-a_{2}\right)^{2 m_{2}-k} \cdots\left(z-a_{s}\right)^{2 m_{s}-k} G(z)}{\left(z-b_{1}\right)^{2 n_{1}+k}\left(z-b_{2}\right)^{2 n_{2}+k} \cdots\left(z-b_{t}\right)^{2 n_{t}+k}}=\frac{Q_{1}(z)}{P_{1}(z)} \text {, say, }
$$

where $P_{1}(z)$ and $Q_{1}(z)$ are also relatively prime polynomials.

If we write $G(z)=\beta\left(z-c_{1}\right)^{l_{1}}\left(z-c_{2}\right)^{l_{2}} \ldots\left(z-c_{q}\right)^{l_{q}}$, where $l_{1}, l_{2}, \ldots, l_{q}$ are non-negative integers; $c_{1}, c_{2}, \ldots, c_{q}$ and $\beta$ are constants with $\beta \neq 0$, and then substitute it into (2.15), then by differentiating (2.15) with the same method as (2.14) follows from (2.11), we can deduce that

$$
\left(f f^{(k)}\right)^{\prime}=\frac{\alpha^{2}\left(z-a_{1}\right)^{2 m_{1}-k-1}\left(z-a_{2}\right)^{2 m_{2}-k-1} \cdots\left(z-a_{s}\right)^{2 m_{s}-k-1} H(z)}{\left(z-b_{1}\right)^{2 n_{1}+k+1}\left(z-b_{2}\right)^{2 n_{2}+k+1} \cdots\left(z-b_{t}\right)^{2 n_{t}+k+1}},
$$

where $H(z)$ is a polynomial with degree at most $\operatorname{deg}(G)+s+t-1$.

We now divide our argument into two cases.

Case 1. If $f f^{(k)}-c$ has exactly one zero, then by (2.15) we may write

$$
f f^{(k)}=c+\frac{\gamma\left(z-z_{0}\right)^{l}}{\left(z-b_{1}\right)^{2 n_{1}+k}\left(z-b_{2}\right)^{2 n_{2}+k} \cdots\left(z-b_{t}\right)^{2 n_{t}+k}}=\frac{Q_{1}(z)}{P_{1}(z)},
$$

where $l$ is a positive integer and $\gamma$ is a non-zero constant. Since $c \neq 0$ we deduce from (2.15) and (2.17) that $a_{\mu} \neq z_{0}$ for $\mu=1,2, \ldots, s$. 
By differentiating (2.17) and noting (2.13) we have

$$
\left(f f^{(k)}\right)^{\prime}=\frac{\gamma\left(z-z_{0}\right)^{l-1} U(z)}{\left(z-b_{1}\right)^{2 n_{1}+k+1}\left(z-b_{2}\right)^{2 n_{2}+k+1} \cdots\left(z-b_{t}\right)^{2 n_{t}+k+1}},
$$

where $U(z)=[l-2 p-k t] z^{t}+e_{1} z^{t-1}+\cdots+e_{t}$ and $e_{1}, \ldots, e_{t}$ are constants.

Next, we shall distinguish two subcases.

Subcase 1.1. If $l \neq 2 p+k t$, then by $(2.17)$ we have $\operatorname{deg}\left(P_{1}\right) \leq \operatorname{deg}\left(Q_{1}\right)$. This together with (2.15) implies that $2 p+k t=\operatorname{deg}\left(P_{1}\right) \leq \operatorname{deg}\left(Q_{1}\right) \leq 2 q-$ $k s+k(s+t-1)$, which leads to $p<q$.

Noting that $a_{\mu} \neq z_{0}$ for $\mu=1,2, \ldots, s$, thus we have from (2.16) and (2.18)

$$
2 q-(k+1) s=\sum_{\mu=1}^{s}\left(2 m_{\mu}-k-1\right) \leq \operatorname{deg}(U)=t .
$$

By this together with $(2.12),(2.13)$, we have $2 q \leq(k+1) s+t \leq \frac{3}{2} q+\frac{1}{2} p$ since $k \geq 2$. This contradicts $p<q$.

Subcase 1.2. If $l=2 p+k t$, then we see from (2.17) that $\operatorname{deg}\left(Q_{1}\right) \leq \operatorname{deg}\left(P_{1}\right)$, and thus by (2.15) we get

$$
2 q-k s+\operatorname{deg}(G)=\operatorname{deg}\left(Q_{1}\right) \leq \operatorname{deg}\left(P_{1}\right)=2 p+k t .
$$

Since $a_{\mu} \neq z_{0}$ for $\mu=1,2, \ldots, s$, again from (2.16), (2.18) we have $l-1 \leq$ $\operatorname{deg}(H)$ and thus

$$
2 p+k t=l \leq \operatorname{deg}(H)+1 \leq \operatorname{deg}(G)+s+t .
$$

Using (2.19), (2.20) and noting (2.12), (2.13), we obtain $2 q \leq k s+s+t \leq \frac{3}{2} q+\frac{1}{2} p$ since $k \geq 2$, which leads to $q \leq p$.

However, if we substitute the fact $\operatorname{deg}(G) \leq k(s+t-1)$ into $(2.20)$, then we have $2 p+k t \leq k(s+t-1)+s+t$, which together with (2.12), (2.13) implies that $2 p \leq k s+s+t-k<\frac{3}{2} q+\frac{1}{2} p$, contradicting $q \leq p$.

Case 2. If $f f^{(k)}-c$ has no zeros, then we see that $l=0$ in equality $(2.17)$ and thus $l \neq 2 p+k t$. By the same proceeding as in the subcase 1.1, we can also get a contradiction.

Hence, $f f^{(k)}-c$ has at least two distinct zeros. Lemma 4 is proved.

Lemma 5. Let $k \geq 3$ be a positive integer and let $c$ be a non-zero constant. If $f$ is a non-constant meromorphic function such that $f$ has only zeros of multiplicity at least $k$ and poles of multiplicity at least 2 , then $f f^{(k)}-c$ has at least two distinct zeros.

Proof. Suppose first that $f$ is a polynomial. Then $f f^{(k)}$ is also a polynomial with degree at least $k$ since $f$ has only zeros of multiplicity at least $k$, and thus $f f^{(k)}-c$ has at least one zero. If $f f^{(k)}-c$ has exactly one zero, say $z_{0}$, then there exist a non-zero constant $\lambda$ and a positive integer $m(\geq k)$ such that $f f^{(k)}=c+\lambda\left(z-z_{0}\right)^{m}$, which, however, must only have simple zero since $c \neq 0$. This is impossible because $f$ is a polynomial and all its zeros have multiplicity at least $k \geq 3$. 
If $f$ is rational but not a polynomial, then Lemma 5 follows from Lemma 4 immediately. Finally, if $f$ is transcendental, then by Lemma 3 we know that $f f^{(k)}-c$ can assume zero infinitely often. The proof of Lemma 5 is complete.

\section{Proof of Theorem 1}

Suppose, to the contrary, that $\mathcal{F}$ is not normal at $z_{0} \in D$. Then by Lemma 1 with $\alpha=k / 2$, there exist points $z_{j} \in D$, functions $f_{j} \in \mathcal{F}$ and positive numbers $\rho_{j}$ such that $z_{j} \rightarrow z_{0}, \rho_{j} \rightarrow 0$ and

$$
g_{j}(\zeta)=\rho_{j}^{-\frac{k}{2}} f_{j}\left(z_{j}+\rho_{j} \zeta\right) \rightarrow g(\zeta)
$$

locally uniformly in $\mathbb{C}$ with respect to the spherical metric, where $g$ is a nonconstant meromorphic function. By Hurwitz's theorem, we see that all zeros of $g$ have multiplicity at least $k$ and all poles of $g$ have multiplicity at least 2 .

On every compact subset of $\mathbb{C}$, we have

$$
f_{j}\left(z_{j}+\rho_{j} \zeta\right) f_{j}^{(k)}\left(z_{j}+\rho_{j} \zeta\right)-c=g_{j}(\zeta) g_{j}^{(k)}(\zeta)-c \longrightarrow g(\zeta) g^{(k)}(\zeta)-c,
$$

spherically uniformly.

If $g g^{(k)} \equiv c$, then $g \neq 0, \infty$ since $c \neq 0$ and thus $c / g^{2}\left(\equiv g^{(k)} / g\right)$ is an entire function. This together with Nevanlinna's first fundamental theorem provides

$$
2 T(r, g)+O(1)=T\left(r, c / g^{2}\right)=m\left(r, c / g^{2}\right)=m\left(r, g^{(k)} / g\right)=S(r, g),
$$

which implies that $g$ is a constant. It is a contradiction. Hence $g g^{(k)}-c \neq 0$.

By Lemma 5 we know that $g g^{(k)}-c$ has at least two distinct zeros, say $\zeta_{0}$ and $\zeta_{0}^{*}$. Thus there exists a positive number $\delta$ and disjoint plane domains $D_{1}$ and $D_{2}$ such that $g g^{(k)}-c$ has no other zeros in $D_{1} \cup D_{2}$ apart from $\zeta_{0}$ and $\zeta_{0}^{*}$, where $D_{1}=\left\{\zeta \in \mathbb{C}:\left|\zeta-\zeta_{0}\right|<\delta\right\}$ and $D_{2}=\left\{\zeta \in \mathbb{C}:\left|\zeta-\zeta_{0}^{*}\right|<\delta\right\}$.

In view of $g g^{(k)}-c \not \equiv 0$, by Hurwitz's theorem and (3.1), we see that there exist points $\zeta_{j} \in D_{1}$ and $\zeta_{j}^{*} \in D_{2}$ such that $\zeta_{j} \rightarrow \zeta_{0}, \zeta_{j}^{*} \rightarrow \zeta_{0}^{*}$ and

(3.2) $f_{j}\left(z_{j}+\rho_{j} \zeta_{j}\right) f_{j}^{(k)}\left(z_{j}+\rho_{j} \zeta_{j}\right)-c=0, \quad f_{j}\left(z_{j}+\rho_{j} \zeta_{j}^{*}\right) f_{j}^{(k)}\left(z_{j}+\rho_{j} \zeta_{j}^{*}\right)-c=0$

for sufficiently large $j$. By the hypotheses of Theorem $1, f_{1} f_{1}^{(k)}$ and $f_{j} f_{j}^{(k)}$ share the value $c$ for all integers $j \geq 2$. It follows from (3.2) that for $j$ large enough

(3.3) $f_{1}\left(z_{j}+\rho_{j} \zeta_{j}\right) f_{1}^{(k)}\left(z_{j}+\rho_{j} \zeta_{j}\right)-c=0, \quad f_{1}\left(z_{j}+\rho_{j} \zeta_{j}^{*}\right) f_{1}^{(k)}\left(z_{j}+\rho_{j} \zeta_{j}^{*}\right)-c=0$.

We now claim that $f_{1} f_{1}^{(k)}-c \not \equiv 0$. Since otherwise we can deduce that $f_{1}$ must be a constant by the same way as we have used above in this section, which contradicts (3.3) and thus our claim is proved. Therefore, the set of all zeros of $f_{1} f_{1}^{(k)}-c$ has no accumulation points. By considering $z_{j}+\rho_{j} \zeta_{j} \rightarrow z_{0}$ and $z_{j}+\rho_{j} \zeta_{j}^{*} \rightarrow z_{0}$, it follows that, for sufficiently large $j, z_{j}+\rho_{j} \zeta_{j}=z_{0}$ and $z_{j}+\rho_{j} \zeta_{j}^{*}=z_{0}$, which leads to $\zeta_{j}=\zeta_{j}^{*}$. This contradicts the fact that $\zeta_{j} \in D_{1}, \zeta_{j}^{*} \in D_{2}$ and $D_{1} \bigcap D_{2}=\varnothing$. Theorem 1 is proved. 


\section{Proof of Theorem 2}

If, to the contrary, $\mathcal{F}$ is not normal at $z_{0} \in D$, then by Lemma 1 with $\alpha=$ $k / 2$, there exist points $z_{j} \in D$, functions $f_{j} \in \mathcal{F}$ and positive numbers $\rho_{j}$ such that $z_{j} \rightarrow z_{0}, \rho_{j} \rightarrow 0$ and $g_{j}(\zeta)=\rho_{j}^{-\frac{k}{2}} f_{j}\left(z_{j}+\rho_{j} \zeta\right)$ converges locally uniformly to a non-constant meromorphic function $g(\zeta)$ in $\mathbb{C}$. By Hurwitz's theorem, all zeros of $g$ have multiplicity at least $k$ and all poles of $g$ have multiplicity at least 2. Clearly $a_{k+2}\left(z_{0}\right) \neq 0, \infty$ since $a_{k+2}(z)$ is holomorphic and non-vanishing in $D$. Hence by Lemma 5 we see that equation $g(\zeta) g^{(k)}(\zeta)+a_{k+2}\left(z_{0}\right)=0$ must have a solution in $\mathbb{C}$.

We may now assume that there exists $\xi_{0} \in \mathbb{C}$ such that $g\left(\xi_{0}\right) g^{(k)}\left(\xi_{0}\right)+$ $a_{k+2}\left(z_{0}\right)=0$. Then $g\left(\xi_{0}\right) \neq \infty$ since $a_{k+2}(z)$ is holomorphic in $D$. So there exists $\delta>0$ such that $g(\zeta)$ is analytic in $D_{2 \delta}=\left\{\zeta \in \mathbb{C}:\left|\zeta-\xi_{0}\right|<2 \delta\right\}$. For $r=1,2, \ldots, k$, since $g_{j}^{(r)}(\zeta) \rightarrow g^{(r)}(\zeta)$ uniformly in $D_{\delta}=\left\{\zeta \in \mathbb{C}:\left|\zeta-\xi_{0}\right|<\delta\right\}$, thus all $g_{j}^{(r)}(\zeta)$ are also analytic in $D_{\delta}$ for sufficiently large $j$. By an elementary computation we have

$$
\begin{aligned}
& g_{j}(\zeta) g_{j}^{(k)}(\zeta) \\
&= f_{j}\left(z_{j}+\rho_{j} \zeta\right)\left\{\sum_{m=0}^{k} a_{m}\left(z_{j}+\rho_{j} \zeta\right) f_{j}^{(k-m)}\left(z_{j}+\rho_{j} \zeta\right)+a_{k+1}\left(z_{j}+\rho_{j} \zeta\right)\right\} \\
&-f_{j}\left(z_{j}+\rho_{j} \zeta\right)\left\{\sum_{m=1}^{k} a_{m}\left(z_{j}+\rho_{j} \zeta\right) f_{j}^{(k-m)}\left(z_{j}+\rho_{j} \zeta\right)+a_{k+1}\left(z_{j}+\rho_{j} \zeta\right)\right\} \\
&= f_{j}\left(z_{j}+\rho_{j} \zeta\right)\left\{\sum_{m=0}^{k} a_{m}\left(z_{j}+\rho_{j} \zeta\right) f_{j}^{(k-m)}\left(z_{j}+\rho_{j} \zeta\right)+a_{k+1}\left(z_{j}+\rho_{j} \zeta\right)\right\} \\
&1) \quad-\sum_{m=1}^{k} a_{m}\left(z_{j}+\rho_{j} \zeta\right) \rho_{j}^{m} g_{j}(\zeta) g_{j}^{(k-m)}(\zeta)-\rho_{j}^{\frac{k}{2}} g_{j}(\zeta) a_{k+1}\left(z_{j}+\rho_{j} \zeta\right),
\end{aligned}
$$

where $g_{j}^{(0)} \equiv g_{j}$. Noting that $\rho_{j} \rightarrow 0, z_{j} \rightarrow z_{0} \in D$ and that for $m=$ $1,2, \ldots, k+1 a_{m}(z)$ are analytic in $D$, thus there exists a constant $L>0$, depending only on $z_{0}$, such that $\left|a_{m}\left(z_{j}+\rho_{j} \zeta\right)\right| \leq L$ for sufficiently large $j$ and $\zeta \in D_{\delta}$. Therefore, we have uniformly $\sum_{m=1}^{k} a_{m}\left(z_{j}+\rho_{j} \zeta\right) \cdot \rho_{j}^{m} \cdot g_{j}(\zeta) g_{j}^{(k-m)}(\zeta) \rightarrow$ 0 in $D_{\delta / 2}=\left\{\zeta \in \mathbb{C}:\left|\zeta-\xi_{0}\right|<\delta / 2\right\}$. By this and (4.1) we have uniformly in $D_{\delta / 2}$

$$
\begin{aligned}
& f_{j}\left(z_{j}+\rho_{j} \zeta\right)\left\{\sum_{m=0}^{k} a_{m}\left(z_{j}+\rho_{j} \zeta\right) f_{j}^{(k-m)}\left(z_{j}+\rho_{j} \zeta\right)+a_{k+1}\left(z_{j}+\rho_{j} \zeta\right)\right\} \\
+ & a_{k+2}\left(z_{j}+\rho_{j} \zeta\right) \\
= & g_{j}(\zeta) g_{j}^{(k)}(\zeta)+\sum_{m=1}^{k} a_{m}\left(z_{j}+\rho_{j} \zeta\right) \rho_{j}^{m} g_{j}(\zeta) g_{j}^{(k-m)}(\zeta)
\end{aligned}
$$




$$
\begin{aligned}
& +\rho_{j}^{\frac{k}{2}} g_{j}(\zeta) a_{k+1}\left(z_{j}+\rho_{j} \zeta\right)+a_{k+2}\left(z_{j}+\rho_{j} \zeta\right) \\
& \longrightarrow g(\zeta) g^{(k)}(\zeta)+a_{k+2}\left(z_{0}\right)
\end{aligned}
$$

It is easy to see that $g(\zeta) g^{(k)}(\zeta)+a_{k+2}\left(z_{0}\right) \not \equiv 0$. If this is not the case, then we can deduce that $g$ is a constant as we have done in Section 3 , a contradiction.

Now we have shown that $g\left(\xi_{0}\right) g^{(k)}\left(\xi_{0}\right)+a_{k+2}\left(z_{0}\right)=0$ and $g(\zeta) g^{(k)}(\zeta)+$ $a_{k+2}\left(z_{0}\right) \not \equiv 0$. Hence by (4.2) and Hurwitz's theorem, there exist points $\zeta_{j}$ such that $\zeta_{j} \rightarrow \xi_{0}$ and

$$
\begin{aligned}
& f_{j}\left(z_{j}+\rho_{j} \zeta_{j}\right)\left\{\sum_{m=0}^{k} a_{m}\left(z_{j}+\rho_{j} \zeta_{j}\right) f_{j}^{(k-m)}\left(z_{j}+\rho_{j} \zeta_{j}\right)\right. \\
& \left.+a_{k+1}\left(z_{j}+\rho_{j} \zeta_{j}\right)\right\}+a_{k+2}\left(z_{j}+\rho_{j} \zeta_{j}\right)=0
\end{aligned}
$$

for sufficiently large $j$. Thus we see from the hypotheses of Theorem 2 that $\left|g_{j}^{(k)}\left(\zeta_{j}\right)\right|=\rho_{j}^{\frac{k}{2}}\left|f_{j}^{(k)}\left(z_{j}+\rho_{j} \zeta_{j}\right)\right| \leq \rho_{j}^{\frac{k}{2}} M$, and thus $\left|g^{(k)}\left(\xi_{0}\right)\right|=\lim _{j \rightarrow \infty}\left|g_{j}^{(k)}\left(\zeta_{j}\right)\right|$ $=0$, which contradicts $g\left(\xi_{0}\right) g^{(k)}\left(\xi_{0}\right)=-a_{k+2}\left(z_{0}\right) \neq 0$. The proof of Theorem 2 is complete.

\section{References}

[1] A. Alotaibi, On the zeros of a $f\left(f^{(k)}\right)^{n}-1$ for $n \geq 2$, Comput. Methods Funct. Theory 4 (2004), no. 1, 227-235.

[2] W. Bergweiler and A. Eremenko, On the singularities of the inverse to a meromorphic function of finite order, Rev. Mat. Iberoamericana 11 (1995), no. 2, 355-373.

[3] M. Buck, A theorem on homogeneous differential polynomials, Results Math. Online First; DOI 10.1007/s00025-012-0234-1(2012).

[4] H. H. Chen and M. L. Fang, On the value distribution of $f^{n} f^{\prime}$, Sci. China, Ser. A. 38 (1995), 789-798.

[5] J. Clunie, On a result of Hayman, J. London Math. Soc. 42 (1967), 389-392.

[6] W. Doeringer, Exceptional values of differetial polynomials, Pacific J. Math. 98 (1982), no. $1,55-62$.

[7] W. K. Hayman, Research Problems in Function Theory, Athlone Press, London, 1967.

[8] X. J. Huang and Y. X. Gu, On the value distribution of $f^{2} f^{(k)}$, J. Aust. Math. Soc. 78 (2005), no. 1, 17-26.

[9] J. K. Langley, The zeros of $f f^{\prime \prime}-b$, Results Math. 44 (2003), no. 1-2, 130-140.

[10] E. Mues, Über ein problem von Hayman, Math. Z. 164 (1979), no. 3, 239-259.

[11] X. C. Pang and L. Zalcman, On theorems of Hayman and Clunie, New Zealand J. Math. 28 (1999), no. 1, 71-75.

[12] J. L. Schiff, Normal Families, Springer, New York, Berlin, Heidelberg, 1993.

[13] J. P. Wang, On the zeros of $f^{n}(z) f^{(k)}(z)-c(z)$, Complex Var. Elliptic Equ. 48 (2003), no. 8, 695-703.

[14] C. C. Yang and P. C. Hu, On the value distribution of $f f^{(k)}$, Kodai Math. J. 19 (1996), no. 2, 157-167.

[15] C. C. Yang, L. Yang, and Y. F. Wang, On the zeros of $f\left(f^{(k)}\right)^{n}-a$, Chin. Sci. Bull. 38 (1993), no. 24, 2125-2128.

[16] C. C. Yang and H. X. Yi, Uniqueness Theory of Meromorphic Functions, Dordrecht, Beijing, New York, Kluwer Academic Publishers and Science Press, 2003.

[17] L. Zalcman, Normal families: New perspectives, Bull. Amer. Math. Soc. (N.S.) 35 (1998), no. $3,215-230$. 
[18] C. P. Zeng, Normality and shared values with multiple zeros, J. Math. Anal. Appl. 394 (2012), no. 2, 683-686.

Department of Mathematics

Shaoxing College of Arts and Sciences

Shaoxing, Zhejiang 312000, P. R. China

E-mail address: jpwang604@hotmail.com 